\title{
Autour des gallicismes contemporains dans le monde hispanophone
}

\begin{abstract}
Résumé
L'importance de l'influence de la langue française sur l'espagnol est un fait incontestable. Son rôle, au cours des siècles écoulés, peut être observé pratiquement à tous les niveaux de la langue : l'orthographe, la phonétique, la morphologie, le lexique, la sémantique, la syntaxe, etc. Cependant, dans les dernières décennies, sa place est progressivement occupée par l’anglais. Néanmoins, le français ne cesse de contribuer à la langue espagnole dans de nombreux processus linguistiques. Ces derniers n'ont pas toujours le même caractère selon les pays hispanophones. Dans cette recherche nous désirons présenter quelques observations sur les apports français à la langue espagnole en tenant compte des différences diatopiques de celle-ci. Nous nous proposons d'atteindre cet objectif en nous basant essentiellement sur des dictionnaires contemporains édités dans différents pays hispanophones. Par conséquent, cette étude aura un caractère, d'une part, lexicographique et lexicologique, et, d'autre part, comparatif. En effet, comme nous allons le démontrer, s'il s'agit des gallicismes, il existe de nombreuses différences dialectales en espagnol, par exemple, au niveau de la prosodie, de l'orthographe, de la morphologie et de la sémantique.
\end{abstract}

Mots-clés : gallicismes, emprunts linguistiques, espagnol américain, typologie des emprunts, dialectologie espagnole

\section{Summary}

The importance of the influence of the French language on Spanish is an indisputable fact. Its role, over the past centuries, can be observed practically at all levels of the language: spelling, phonetics, morphology, vocabulary, semantics, 


\section{Piotr SORBET}

syntax, etc. However, in recent decades, its place is gradually occupied by English. Nevertheless, French does not cease to contribute to the Spanish language in many linguistic processes. The interferences don't have always the same character according to the Spanish-speaking countries. In this research we propose to present some observations on the French contributions to the Spanish language taking into account the diatopic differences of this one. We want to achieve this goal based mainly on contemporary dictionaries published in various Hispanic countries. Consequently, this study will have, on one hand, a lexicographic and lexicological character, and on the other a comparative purpose. Indeed, as we will demonstrate, in the Spanish Gallicisms there are many dialectal differences, for example, in terms of prosody, spelling, morphology and semantics.

Keywords: Gallicisms, linguistics borrowing, American Spanish, typology of loans, Spanish dialectology

\section{Introduction}

Dans les dernières décennies l'essor de l'impact de l'anglais, principalement états-unien mais aussi britannique, sur l'espagnol a conduit l'attention de nombreux linguistes vers les anglicismes. Les innombrables dictionnaires, articles et autres travaux scientifiques où l'on décrit les éléments anglais, bien qu'ils restent sur certains aspects incomplets, sont de plus en plus exhaustifs et satisfaisants. Pour ce qui est des études analogues qui visent à décrire les apports contemporains de la langue française à l'espagnol, celles-ci sont, d'un côté, moins nombreuses, et d'un autre côté, dans beaucoup de cas, elles se concentrent essentiellement sur l'espagnol européen en laissant à part, par exemple, le continent américain. Ce manque de travaux détaillés a été déjà signalé, à plusieurs reprises, par différents linguistes (Cahuzac, 1988 : 127 ; Corbella, 1996/97 : 569; Sala, 1982 : 431). Les recherches consacrées à l'analyse des gallicismes en Amérique sont la plupart du temps concises. Souvent, elles se limitent à une variante diatopique et dans la plupart des cas ce sont des articles de valeur mais ce ne sont pas des contributions approfondies. Ceci n'est pas sans raisons. En premier lieu, comme nous l'avons déjà signalé, il s’agit de la prépondérance de l'anglais dans les études à propos d'emprunts distincts où on restreint l'importance du français. En deuxième lieu, la description de l'influence du français sur une langue telle que l'espagnol, qui est fortement diversifiée dialectalement, requiert l'assemblage de multiples dictionnaires et travaux de divers pays, ce qui fait que le corpus 
lexicographique-lexicologique potentiel est démesuré. En troisième lieu, nous sommes persuadé qu'une recherche qui permettrait d'exposer les gallicismes qui fonctionnent en espagnol et qui prendrait en considération les différences diatopiques nécessite, d'une part, une méthodologie appropriée, et, d'autre part, une typologie qui permettrait de classer adéquatement les éléments d'origine hexagonale. Pour cette raison, nous voulons proposer dans cette contribution une classification des apports français à la langue espagnole en tenant compte de la variation diatopique de celle-ci.

\section{Cadre théorique}

Un gallicisme est un emprunt d'origine française qui fonctionne dans un autre idiome. Cette définition ne présente pas de difficultés. Néanmoins, la description de son hyperonyme, c'est-à-dire de l'emprunt linguistique, suscite d'incessantes polémiques. Il y en a qui l'identifient à un mot (Tagliavini, 1993 [1973] : 368) et d'autres qui le définissent comme un élément (Deroy, 1956: 2 ; Sorbet, 2016c : 101). En même temps, certains essaient de proposer des descriptions plus complexes dans lesquelles il est identifié avec un processus :

«Processus par lequel une langue L1 dont le lexique est fini et déterminé dans l'instant T acquiert un mot M2 (expression et contenu) qu'elle n'avait pas et qui appartient au lexique d'une langue L2 (également fixe et déterminé dans l'instant T). Ce processus se déroule de l'instant T à l'instant T', le temps écoulé entre $\mathrm{T}$ et $\mathrm{T}$ ' est très variable et correspond à la codification plus ou moins rapide d'un élément de discours dans la langue.» (Rey-Debove, 1973 : 107).

Observons que cette définition restrictive concerne seulement l'emprunt lexical et laisse à part les emprunts d'autres types : sémantiques, phonétiques, orthographiques, prosodiques, etc. De plus, elle ne permet pas de discerner le contraste entre le résultat et le processus au cours duquel on emprunte une unité linguistique. Elle maintient, alors, à cet égard, l'ambiguïté commune à toutes les langues romanes (Esp. Gal. préstamo, It. prestito, Port. empréstimo, Roum. imprumut, etc.) qui ne distinguent pas ces deux notions, à la différence des langues slaves qui expriment cette dichotomie en se servant de deux termes. Par exemple, en polonais zapożyczenie désigne le résultat de zapożyczanie, c'est-àdire du processus de l'emprunt (Sorbet, 2014a : 98 ; 2016c : 110). Il est donc plus convenable d'interpréter l'emprunt comme une reproduction / représentation 
d'un modèle étranger (Haugen, 1950 : 212). Le modèle, étant un terme suffisamment général tout en étant précis, peut concerner naturellement, à notre avis, tous les niveaux de la langue. Par conséquent, il est possible de distinguer des modèles lexicaux, phonétiques, orthographiques, prosodiques, morphologiques, syntaxiques, sémantiques inhérents à une langue qui passent dans un autre système linguistique. Si ces modèles sont d'origine française ou anglaise nous parlons, alors, respectivement, de gallicismes ou d'anglicismes.

\subsection{Gallicismes espagnols}

L'histoire de l'influence de la langue française sur l'espagnol remonte au Moyen Âge. C'est à cette époque que Sancho le Grand, roi de Castille, au XIe siècle a tracé une nouvelle route (Esp. camino francés), moins dangereuse et moins pénible qui conduisait à Saint-Jacques-de-Compostelle. Ceci ainsi que les réformes clunisiennes et cisterciennes ont incité des immigrés venus de l'Hexagone à s'établir dans la Péninsule Ibérique, ce qui a permis à de multiples mots d'origine française et provençale d'entrer dans la langue espagnole : deán " doyen ecclésiastique», homenaje "hommage», mensaje « message», mesón « auberge", pitanza « ration", viandas « mets» (Sorbet, $2015: 82$ ). Au cours des siècles suivants, le rôle du français a augmenté jusqu'à atteindre son apogée aux dix-huitième et dix-neuvième siècles quand son influence était si grande que de nombreux linguistes et intellectuels, des deux côtés de l'Atlantique, ont essayé de le freiner en publiant des travaux de divers types où on décrivait et on condamnait une grande partie des gallicismes. Le premier à éditer un dictionnaire de ce type a été Rafael María Baralt, poète, historien et écrivain d'origine vénézuélienne, qui a publié son Diccionario de Galicismos en 1855. Il a réuni alors des centaines de mots et de constructions qui, selon l'auteur, devaient être évités et remplacés par des équivalents autochtones. Ceci s'inscrivait dans les tendances puristes qui proliféraient à cette époque chez une grande partie des intellectuels hispanophones 1 . Ces efforts ont parfois échoué, mais dans d'autres cas ils ont fini par éliminer beaucoup d'emprunts d'origine

1 Observons que ceci peut être comparé, jusqu’à un certain degré, à la situation contemporaine dans la Belgique septentrionale où le purisme linguistique cherche à remplacer successivement les emprunts au français par des mots néerlandais : franc. parapluie $>$ néerl. de paraplu $>$ de regenscheren, franc. centrifugeuse $>$ néerl. de centrifuge $>$ de droogzwierder, franc. punaise $>$ néerl. de punaise $>$ de duimspijker (Prędota, $2003: 45)$. 
française. En tout cas, il y a beaucoup de gallicismes dans différents pays hispanophones qui sont tombés en désuétude ou sont aujourd'hui rarement utilisés, et ils ne figurent plus dans les dictionnaires. Ainsi en République Dominicaine au XVIII siècle on utilisait : furnida «id. » < franc. (cheveux) fournis, gardami " au secours" < franc. garde à moi, pasible « id. » < franc. paisible, sursa / zurza « source d'eau » < franc. source (cf. Granda, 1987-1989; 1990); en Espagne aux XVI e et XVIIe siècles redán « id. » < franc. redan, pitiflor « id. » < franc. petite fleur, donjón «id. »< franc. donjon, fripería «id. »< franc. friperie, embrazura «id.» < franc. embrasure (Varela Merino, 2009 : s. v.) ; en Argentine au XVIII siècle : citoyén " sorte de capote» < franc. citoyen (Fontanella de Weinberg, 1986 : 88) et au XIX ${ }^{\mathrm{e}}$ siècle degringolade " chute, culbute, ruine» < franc. dégringolade, enquete «id. » < franc. enquête, vesindaje "id. » < franc. voisinage (Garzón, 1910 : s. v.), etc.

L'impact du français varie, donc, selon les époques et les aires dialectales. Les gallicismes contribuent, par conséquent, à la différenciation diatopique de l'espagnol car ils font partie, simultanément, d'une part, des américanismes (americanismos), et, d'autre part, des espagnolismes (españolismos). Nous allons présenter ces deux notions plus en détail ci-après.

\subsection{Américanisme et espagnolisme}

Bien que la définition d'américanisme soit sujette à de nombreuses polémiques parmi les spécialistes (cf. Bohórquez, 1994), nous partirons du principe selon lequel un mot pour être qualifié d'américanisme doit manifester une différence quel que soit son caractère ou son origine face à l'espagnol péninsulaire. Voici quelques exemples lexicaux :

\begin{tabular}{|c|c|c|}
\hline Espagnol américain & Espagnol européen & signification \\
\hline plata & dinero & "argent » \\
papa & patata & "patates » \\
cortina de hierro & telón de acero & "rideau de fer » \\
carpa & tienda de campaña & "tente » \\
altoparlante & altavoz & «haut-parleur » \\
\hline
\end{tabular}

L'américanisme a de multiples hyponymes, entre autres, les argentinismos 'argentinismes', mexicanismos 'mexicanismes', cubanismos 'cubanismes' et peruanismos 'péruvianismes'. À côté de ceux-ci, par analogie, il faut, bien 


\section{Piotr SORBET}

entendu, discerner les filipinismos 'philippinismes', les ecuatoguineanismos 'équatoguinéismes' et les luísianismos [Lu] 'louisianismes'. Notons que, dans tous ces cas, il s'agit d'opposer l'espagnol européen aux variantes qui sont utilisées en dehors du Vieux Continent. Tous ces termes ont un caractère restrictif car ils indiquent une limitation géographique. Méthodologiquement, ils devraient être confrontés, selon certains linguistes, par exemple Lope Blanch (1995 : 440), à l'espagnolisme (españolismo) qui aurait le sens de "particularité lexicale, grammaticale ou syntaxique du parler des Espagnols». Néanmoins, pour le moment, ce vocable bien qu'il soit répertorié dans le Diccionario de la lengua española (DEL, consulté le 30.09.2016), ne possède pas cette acception, et dans son deuxième sens il renvoie à l'article hispanismo :

\begin{tabular}{|c|c|}
\hline $\begin{array}{l}\text { españolismo } \\
\text { 1. m. Cualidad o condición de español. } \\
\text { 'Qualité ou trait de caractère epsagnol.' } \\
\text { 2. m. hispanismo. 'hispanisme.' } \\
\text { 3. m. Amor o apego a lo español. 'Amour } \\
\text { ou attachement à ce qui est espagnol.' }\end{array}$ & $\begin{array}{l}\text { hispanismo } \\
\text { 1. m. Giro o modo de hablar propio y pri- } \\
\text { vativo de la lengua española. 'Tournure } \\
\text { ou façon de parler propre et privative de } \\
\text { la langue espagnole.' } \\
\text { 2. m. Vocablo o giro de la lengua españo- } \\
\text { la empleado en otra. 'Mot ou tournure } \\
\text { de la langue espagnole utilisé dans une } \\
\text { autre langue.' } \\
\text { 3. m. Empleo de vocablos o giros es- } \\
\text { pañoles en distinto idioma. 'Emploi de } \\
\text { mots ou tournures espagnols dans une } \\
\text { différente langue.' } \\
\text { 4. m. Afición al estudio de las lenguas, } \\
\text { literaturas o cultura hispánicas. 'Goût } \\
\text { pour l'étude des langues, littératures ou } \\
\text { culture espagnoles.' }\end{array}$ \\
\hline (DRAE, consulté le 30.09.2016) & \\
\hline
\end{tabular}

L'inclusion de l'entrée de ce sens du terme españolismo dans les dictionnaires généraux créerait, à notre avis, un certain parallélisme dans la terminologie lexicologique de l'espagnol. Ceci faciliterait aussi la description de nombreux termes qui sont utilisés en même temps en Espagne et dans un ou plusieurs pays hors-européens mais qui ne sont pas, par exemple, des panaméricanismes.

En ce qui concerne la présence des éléments français en espagnol, ceuxci peuvent avoir un caractère panhispanique [Panhi] (Espagne, Amérique, 
Afrique, Asie), panaméricain [PanA] (l'ensemble ou la quasi-totalité de l'Amérique) ou régional (plusieurs pays, un pays, une région). Dans certains cas il s'agit aussi d'une influence dispersée dans des zones sans contiguïté géographique. Par conséquent, nous sommes persuadé qu'il existe, entre autres, des gallicismes panhispaniques, panaméricains, des régions du Rio de la Plata, du Cône Sud, des Andes, argentins, mexicains, colombiens, louisianais, etc.

Enfin, il faut insister sur le fait que souvent les différences qui opposent les dialectes de l'espagnol, par exemple, ceux des deux côtés de l'Océan Atlantique, sont dues aux interférences linguistiques du français et de l'anglais. En effet, ce dernier, naturellement, a pu exercer son influence plus fortement dans les pays hispano-américains. Ainsi, il est possible de discerner deux modèles linguistiques : le français et l'anglais, qui expliquent de nombreuses différences lexicales, par exemple:

\begin{tabular}{|c|c|}
\hline $\begin{array}{l}\text { Modèle français : Espagne } \\
\text { (espagnolismes) }\end{array}$ & $\begin{array}{l}\text { Modèle anglo-américain : } \\
\text { Hispanoamérique } \\
\text { (américanismes) }\end{array}$ \\
\hline ordenador $<$ franc. ordinateur & $\begin{array}{l}\text { computer : États-Unis [EU], Mexique } \\
\text { [Mx], Guatemala [Gu], Honduras [Ho], } \\
\text { Salvador [ES], Nicaragua [Ni], Panama } \\
\text { [Pa], République Dominicaine [RD], Ve- } \\
\text { nezuela [Ve], Pérou [Pe], Chili [Ch] ; [EU, } \\
\text { Mx, Gu, Ho, ES, Ni, RD, Ve, Pe] Cuba } \\
\text { [Cu], Puerto Rico [PR], Bolivie [Bo], Pa- } \\
\text { raguay [Py], Argentine [Ar], Uruguay [Ur] } \\
\text { computadora < ang. computer; [EU, } \\
\text { Mx, Gu, Ho, ES, Ni, Pa, RD, Ve, Pe, Ch] } \\
\text { computador (DASALE) }\end{array}$ \\
\hline horas punta < franc. heures de pointe & $\begin{array}{l}\text { horas pico: [Mx, Pa, RD, Ve, Pe, Bo, } \\
\text { Ch, Py, Ar, Ur], Équateur [Ec], Colombie } \\
\text { [Co], Costa Rica [CR] < ang. peak hours } \\
\text { (DASALE) }\end{array}$ \\
\hline $\begin{array}{l}\text { Asuntos exteriores < franc. Affaires } \\
\text { étrangères }\end{array}$ & $\begin{array}{l}\text { Relaciones exteriores < ang. Foreign } \\
\text { Relations }\end{array}$ \\
\hline parachoques $<$ franc. pare-chocs & $\begin{array}{l}\text { bómper [Gu, Ho, ES, Ni, Pa, RD, PR, Co] } \\
\text { < ang. bumper (DASALE) }\end{array}$ \\
\hline
\end{tabular}




\section{Piotr SORBET}

Bien entendu, l'existence des deux modèles en question n'est pas suffisante pour pouvoir éclaircir les causes de toutes les différences lexicales entre les deux continents. En effet, pour les exposer, chaque pays mériterait une contribution qui dépasserait largement l'espace d'un article comme celui-ci.

\section{Corpus}

Comme les données qui nous intéressent sont très dispersées dans des dizaines voire des centaines de sources lexicographiques, nous avons décidé, pour cette recherche, de réunir une série de dictionnaires qui vont constituer notre point de départ. Ceux-ci, au fur et à mesure des futures contributions, vont être successivement complétés par d'autres œuvres et travaux édités dans différents pays hispanophones. Les dictionnaires que nous avons pris en considération sont les suivants : Diccionario de uso del español de Chile (DUECh), Diccionario integral del español de la Argentina (DIEA), Diccionario de americanismos (DASALE), Diccionario del español de Argentina (DEAr), Nuevo diccionario de uruguayismos (DEUr), Nuevo diccionario de colombianismos (DECo), Diccionario de salvadoreñismos (DEES), Diccionario de venezolanismos (DEVe), Diccionario del español usual en México (DEUMx), Diccionario etimológico del lunfardo (DELOC), ainsi que les dictionnaires généraux : le Diccionario de la lengua española (DLE) et le Diccionario de Uso del español de María Moliner (DUE). Nous nous sommes servi également de certains travaux métalinguistiques que nous indiquons dans la bibliographie à la fin du présent article.

Comme il est possible de le constater, la répartition diatopique et diastratique des œuvres consultées n'est pas tout à fait équilibrée. Certaines variantes sont surreprésentées par rapport aux autres et certaines sont omises. Pour cette raison, dans ce travail, nous ne prétendons pas fournir des données quantitatives mais qualitatives. En effet, notre but principal est de proposer une typologie des gallicismes en espagnol contemporain.

\section{Gallicismes contemporains dans le monde hispanophone}

Eu égard au nombre des emprunts hispaniques au français ces derniers peuvent être classifiés de plusieurs manières. Dans la division ci-dessous nous présenterons les gallicismes hispaniques en fonction de la catégorie 
d'emprunt où il est possible de les répertorier en tenant compte des différences dialectales conformes au cadre théorique présenté plus haut. Nous n'allons pas, alors, nous occuper minutieusement, à cette occasion, des éléments français qu'on utilise pareillement dans l'ensemble du monde hispanophone.

Pour ce qui est des indications géographiques nous avons pris comme référence essentiellement le DASALE. Cependant ces informations devraient être interprétées comme approximatives. De plus, vu que les données fournies par les sources sont souvent contradictoires, les autres marques et informations telles que 'archaïsme', 'désuet', 'populaire', 'familier', 'obsolète' seront omises. Les groupes principaux des gallicismes lexicaux-sémantiques que nous allons exposer sont les suivants : formels et sémantiques (3.1.1.), sémantiques (3.1.2.), calques structuraux (3.1.3.). Puis nous présenterons certaines caractéristiques différenciatives (3.2.) qui permettent de discerner des dissemblances dialectales supplémentaires dans le cadre des emprunts d'origine française.

\subsection{Gallicismes lexicaux-sémantiques}

Cette catégorie comprend, avant tout, les gallicismes, d'un côté, formels et sémantiques (3.1.1.), et d'un autre côté, seulement sémantiques (3.1.2.). De plus, nous sommes convaincu qu'il est également nécessaire d'évoquer un troisième groupe, dit intermédiaire entre les deux précédents, les calques structuraux (3.1.3).

\subsubsection{Gallicismes formels et sémantiques}

Sans vouloir offrir une liste exhaustive indiquons : franc. cache-nez $>[\mathrm{Mx}]$ gasné, gazné « cravate ou foulard pour homme » (DASALE); franc. fouet $>[\mathrm{Mx}$, $\mathrm{Gu}, \mathrm{Ho}, \mathrm{ES}, \mathrm{Ni}, \mathrm{Pa}, \mathrm{Cu}, \mathrm{RD}, \mathrm{PR}, \mathrm{Co}, \mathrm{Ve}, \mathrm{Ec}, \mathrm{Pe}$; Bo, CR] fuete (DASALE, DECo, $\mathrm{DECu}, \mathrm{DEES}, \mathrm{DEVe}, \mathrm{DEUMx}$ ), [Mx, Ho, Cu, RD, Ec, Pe] foete (DASALE), $[\mathrm{PR}]$ juete «id. » (DASALE) ; franc. garçon > [Ch, Bo, Ec] garzón « serveur » (DASALE, DUECh); franc. décoller $>$ [Co, Ec, Ar, Ur; Ho, Ch] decolar « quitter le sol, en parlant d'un avion » (DASALE, DEAr, DECo) ; franc. gamin > [Co, Ve, Ec] gamín « enfant vagabond» (DASALE); franc. briquet $>[\mathrm{Co}]$ briqué «id. » (DASALE, DECo) ; franc. brassière > [EU, Mx, Gu, Ho, ES, Ni, CR, Pa, $\mathrm{Cu}, \mathrm{RD}, \mathrm{PR}, \mathrm{Co}, \mathrm{Ve}, \mathrm{Ec}, \mathrm{Pe}, \mathrm{Bo}, \mathrm{Ch}, \mathrm{Py}, \mathrm{Ur}$ ] brasière (DASALE), [EU, Ho, RD, $\mathrm{PR}, \mathrm{Bo}, \mathrm{Py}, \mathrm{Ur}, \mathrm{Cu}$ ] brassiere (DASALE, DECu), [ES] brasiel (DEES), [Ni] bra- 
zier « id. » (DASALE, DECo, DECu, DEES), [EU, Mx, Gu, Ho, ES, Ni, CR, Pa, $\mathrm{Cu}, \mathrm{RD}, \mathrm{PR}, \mathrm{Co}, \mathrm{Ve}, \mathrm{Ec}, \mathrm{Pe}, \mathrm{Bo}, \mathrm{Ch}, \mathrm{Py}, \mathrm{Ur}]$ brasier (DASALE, DEES, DEUMx), [Mx, Ho, ES, Ni, CR, RD] brassier (DASALE, DEUMx); franc. soutien-gorge $>$ [Ar] sutién, [Co] sutián « id. »; franc. vitrine > [Ho, ES, Ni, CR, Pa, RD, PR, $\mathrm{Co}, \mathrm{Ec}, \mathrm{Bo}, \mathrm{Ch}, \mathrm{Py}$.$] vitrina « devanture vitrée d'un local commercial » (DA-$ SALE, DECo] ; franc. bétonnière > [Ch] betonera «id. » (DASALE, DEUCh); franc. usine > [Co, Ec, Pe, Bo, Ch, Py, Ar, Ur.] usina « id.» (DASALE, DUECh); franc. exploser $>[\mathrm{Ch}]$ explosar «id.» (DASALE); franc. monsieur $>[\mathrm{Ve}]$ musiú « étranger» (DEVe, DASALE), franc. bretelle > [RD, Ch, Ar, Ur] «id. » (DASALE, DEAr, DEUr).

\subsubsection{Gallicismes sémantiques}

Ces emprunts au français concernent principalement le niveau sémantique. Recensons à titre d'exemple : franc. accorder $>$ [RD, Py, Ar, Ur.] acordar « accorder (attribuer)» (DASALE, DEAr, DEUr) ; franc. addition > [Ar, Ur] adición «id. » (DASALE, DEAr, DELOC, DEUr); franc. poule > [Pa, Ec, Pe, Bo, Py, Ar, Ur.] polla « enjeu » (DASALE, DEAr); franc. tenue $>$ [Pe, Bo, Ch] tenida «id. »; franc. (pince-)monseigneur $>$ [Ar] arzobispo «id. » (DELOC); franc. affecter > [Ve, Ar, Ur] afectar « affecter (destiner) » (DASALE, DIEA, DEAr, DEUr).

\subsubsection{Calques structuraux}

D’après Sala (1982: 248), c'est en Argentine et en Colombie qu'on peut trouver le plus de gallicismes de ce type. Cependant, les traductions partielles et les calques complets existent dans toutes les variantes de l'espagnol. Recensons : franc. chemin de fer $>$ [Lu] camino de hierro «id. » (Pratt, $2004: 150)$; franc. fermeture éclair $>$ [Ve, Ec, Pe, Bo, Py, Ar, Ur.] cierre relámpago (DASALE, DEAr, DEUr), [Ch] cierre eclair « id. » (DUECh); franc. vase de nuit $>$ [Ve, Pe; Co] vaso de noche «id. " (DASALE); franc. longue-vue > [Ni, Pa, Ve, $\mathrm{Pe}, \mathrm{Bo}, \mathrm{Ch}, \mathrm{Ar}, \mathrm{Ur}$; Py] largavista(s) «id. » (DASALE, DEAr); franc. médecin légiste > [Mx, RD, Co, Ec, Bo, Ch, Py, Ur, Ar] médico legista «id. " (DASALE, DECo, DUECh, Mx, Ho, ES, Ni, CR, RD.) ; franc. gendarme couché > [Ho, $\mathrm{Ni}, \mathrm{Cu}, \mathrm{RD}, \mathrm{PR}, \mathrm{Co}, \mathrm{Ve}, \mathrm{Ec}$.$] policía a costado (DASALE, DECo, DECu) ; [CR]$ policía dormido « ralentisseur» (DASALE); franc. lettre recommandée $>[\mathrm{Pa}$, Co, Ur] carta recomendada «id. »(DASALE, DECo). 


\subsection{Caractéristiques différenciatives}

Comme nous venons de l'illustrer sommairement, l'utilisation des gallicismes peut être restreinte diatopiquement. Cependant, les fonctionnements de ces emprunts peuvent également se différencier au niveau de la sémantique (3.2.1.), de la fréquence d'emploi (3.2.2.), de la prosodie (3.2.3.), de l'orthographe et de la phonétique (3.2.4.), des catégories grammaticales (3.2.5.), de la morphologie (3.2.6.) et de la productivité (3.2.7.). Tous ces facteurs constituent des caractéristiques différenciatives aussi bien des emprunts d'origine française que des variantes régionales du monde hispanophone.

\subsubsection{Acceptions particulières}

Parmi les gallicismes lexicaux-sémantiques il y en a beaucoup qui, selon les variantes, ont des significations particulières. Ces dernières sont dues tantôt à l'influence française tantôt à l'évolution interne de l'espagnol de ces mots. Mentionnons: franc. vitrine> vitrina [Panhisp] «meuble vitré », [Ho, ES, Ni, $\mathrm{CR}, \mathrm{Pa}, \mathrm{RD}, \mathrm{PR}, \mathrm{Co}, \mathrm{Ec}, \mathrm{Bo}, \mathrm{Ch}, \mathrm{Py}]$ « devanture vitrée d'un local commercial » (DASALE, DECo), [Ve] « aire de supériorité »; franc. garage > garaje [Panhisp] «garage (abri)» (DLE, DUE), [Panhisp] «garage (atelier)» (DLE), [Cu, PR] «station d'essence» (DASALE, DECu, DRAE); franc. cadet > cadete [Panhisp] «gentilhomme qui apprenait les métiers des armes » (DRAE, DUE), [Py, Ar, Ur; $\mathrm{Ch}$ ] « apprenant dans un établissement commercial» (DASALE, DEAr).

\subsubsection{Fréquence d'emploi}

Le fonctionnement des gallicismes peut varier selon leur fréquence d'emploi. Ainsi franc. affiche > affiche, afiche «id. » (DEAr, DECu, DEUr, DEVe); franc. voyeur > voyeurista «id. », bien qu'ils soient connus en Espagne, sont utilisés davantage en Amérique Latine (DUE, DASALE). Ce sont, alors, des gallicismes américains $[\mathrm{Pan} \mathrm{A}]$ de fréquence d'emploi.

\subsubsection{Prosodie}

Les lexies de ce groupe ont différentes accentuations selon les pays: franc. bébé> [Esp] bebé (DUE, DLE), [Gu, Ho, PR, Ec, Pe, Py, Ar, Ur] bebe (DASALE, DIEA); franc. chiffon $>$ [Esp] chiffon (DUE), [Ar] chifón, chiffón (DIEA) ; franc. chauffeur $>$ [Esp] chófer (DUE, DLE) ; [Mx, Gu, Ho, ES, Ni, CR, Pa, Cu, RD, PR, Co, 


\section{Piotr SORBET}

Ve, Ec, Pe, Bo, Ch, Py, Ar, Ur.] chofer (DASALE, DECu, DEUMx); franc. châssis $>$ [Esp] chasis (DUE, DLE), [Mx, Gu, ES, CR, Pa, Co, Ve, Ec, Pe, Bo, Ch, Py, Sv] chasís (DASALE, DECo, DEES).

\subsubsection{Caractéristiques orthographiques et phonétiques}

Les emprunts espagnols au français en plus de la prosodie qui varie, parfois, en fonction des zones géographiques (cf. 3.2.3), peuvent aussi avoir des graphies et/ou des prononciations différentes selon les pays hispanophones. Citons : franc. garage $>$ [Esp] garaje [garáxe], [Ar] garage [garáš(e)] (DEAr, DIEA); franc. bricolage $>$ [Esp] bricolaje [bricoláxe], [Ar] [bricoláy] (DIEA); franc. élite $>$ [Esp] elite [elite] (DLE, DUE), [RD] elit (DASALE) ; franc. pyjama > [Esp] pijama [pixáma], [Mx, Ho, Ni, CR, Cu, RD, PR, Co, Ve, Ec, Pe, Bo, Py, Ar, Ur; Ch] piyama (DASALE, DEUMx) ; franc. bidet > bidet, bidé (DLE, DUE), [Co] bidel (DECo).

\subsubsection{Au niveau du genre grammatical}

Les mots espagnols d'origine française n'ont pas toujours le même genre grammatical que leurs étymons : franc. f. affiche $>$ [Gu, Ho, ES, Ni, CR, Pa, Cu, RD, PR, Co, Ve, Ec, Pe, Bo, Ar, Ur; Mx, Esp] m. afiche (DASALE, DECu, DEUr); franc. f. bretelle $>[\mathrm{RD}, \mathrm{Ch}, \mathrm{Ar}, \mathrm{Ur}] \mathrm{m}$. bretel (DASALE, DEAr). En plus, le genre de la forme espagnole peut ne pas être pareil selon les régions: franc. manucure $>$ [EU, Ho, $\mathrm{Ni}, \mathrm{Pa}, \mathrm{Cu}, \mathrm{PR}, \mathrm{Co}, \mathrm{Ve}, \mathrm{Pe}, \mathrm{Bo}, \mathrm{Ch}$ f. manicure, [Mx, ES, CR, Pa, Ec.] m. manicure, [Sv, Ni, RD, Ve, Bo, Ar] maniquiur (DASALE, DEES); franc. coupé $>$ [Esp] m. cupé, [Ar] f. cupé(DIEA). Parfois la différence de genre consiste dans le fait que dans une partie du monde hispanophone on oppose la variante féminine à la variante masculine : franc. m. bébé $>[\mathrm{Esp}] \mathrm{m}$. bebé, [Gu, Ho, PR, Ec, Pe, Py, Ar, Ur.] m., f. bebe, - a (DASALE, DIEA) ; franc. m. cadet. $>$ [Esp] m. cadete, [Ar] m., f. cadete, $-a$ (DASALE, DIEA).

\subsubsection{Morphologie}

Le radical et le sens du gallicisme sont les mêmes mais les mots sont morphologiquement distincts. Il s'agit, le plus souvent, d'une terminaison différente : franc. grippe $>[\mathrm{Mx}, \mathrm{Pa}, \mathrm{Co}, \mathrm{Ec}$.] gripa (DASALE, DECo, DEUMx), [ES] gripola (DASALE, DEES), [Esp] gripe; franc. manucure $>$ [Esp] manicura, [EU, Ho, Ni, $\mathrm{Pa}, \mathrm{Cu}, \mathrm{PR}, \mathrm{Co}, \mathrm{Ve}, \mathrm{Pe}, \mathrm{Bo}, \mathrm{Ch}, \mathrm{Mx}, \mathrm{ES}, \mathrm{CR}, \mathrm{Pa}, \mathrm{Ec}$.] manicure (DASALE) ; franc. alevin $>$ [Esp] alevín, [Bo, Ar, Ur; Co, Ch] alevino (DASALE); franc. bordeaux 
$>$ [Esp] burdeos, [Ar, Ur] bordeaux « couleur rouge foncé » (DASALE, DIEA, DEAr, DEUr) ; franc. betterave $>$ [Ar, Bo] beterava (DASALE), [Ch] betarraga (DASALE, DEUCh), [Pe, Bo, Ch] beterraga « id.» (DASALE, DEUCh).

\subsubsection{Productivité}

Les gallicismes lexicaux-sémantiques deviennent, souvent, la base de nombreuses formes nouvelles. En premier lieu, il s'agit des dérivés : franc. affiche $>$ esp. afiche $\rightarrow[\mathrm{RD}, \mathrm{Ec}]$ afichar " poser des affiches » (DASALE), [ES] afichero « afficheur» (DASALE, DEES) ; franc. boulon > [Ho, Ni, Ar, Ur.] bulón " grande vis à tête arrondie » $\rightarrow$ [Ar] abulonado (DASALE), [Ar, Py] abulonar (DASALE), [Ar] bulonera (DEAr, DIEA), [Ar, Ur] bulonería (DEAr, DIEA), [Ar, Ur] bulonero (DIEA, DELOC), [Ar] bulonazo (DELOC). En deuxième lieu, les emprunts français se transforment en une partie de multiples expressions idiomatiques qui sont utilisées seulement dans certaines zones hispanophones: franc. monsieur $>[\mathrm{Ve}]$ hacerse el musiú « faire la sourde oreille; simuler l'ignorance » (DASALE, DEVe); franc. usine $>[\mathrm{Bo}, \mathrm{Py}, \mathrm{Ar}, \mathrm{Ur}]$ usina de rumores « milieu où ont fait courir des rumeurs, cancans" (DASALE); franc. couche $>[\mathrm{Ho}]$ ser la cuja « être fainéant» (DASALE); franc. boulon > [Ar, Ur] comer bulones « ingérer des aliments difficiles à digérer » (DASALE, DEAr), [Ar] «se dit d'une personne physiquement forte ». En troisième lieu, les gallicismes, après avoir expérimenté la vesreïsation deviennent, quelquefois, des vesreïsmes : franc. chauffeur $>[\mathrm{Pe}, \mathrm{Bo}$, Ar] chofer $\rightarrow$ [Pe, Bo, Ar] fercho, - a (DASALE, DELOC) ; franc. bouteille $>$ [Panhisp] botella $\rightarrow$ [Ar, Ur] llatebo « bouteille, surtout en verre et pour une boisson alcoolique » (Sorbet, 2014b, 2016a, 2016b). Bien entendu, souvent ce ne sont pas des gallicismes proprement dits. Cependant, aussi bien les dérivés que les expressions figées, d'une part, favorisent l'enracinement des gallicismes en espagnol, et, d'autre part, contribuent à la différenciation des variantes espagnoles.

\section{En guise de conclusion : perspectives de futures recherches}

Nous avons examiné les types les plus importants des gallicismes hispaniques dans le cadre du vocabulaire. Il faut souligner, néanmoins, que certains d'entre eux appartiennent simultanément à deux ou plusieurs des groupes que nous avons évoqués. Nous avons également tracé quelques caractéristiques différenciatives qui permettent de discerner les utilisations propres à certaines aires 


\section{Piotr SORBET}

dialectales des emprunts espagnols. Ainsi pensons-nous être parvenu à montrer de nombreuses différences repérables dans l'utilisation des gallicismes hispaniques.

Cependant, nous croyons que chaque variante, bien que ceci soit un travail de Sisyphe, mériterait une étude approfondie indépendante. Il s'agirait aussi, alors, de décrire les variantes qui constituent assez rarement le centre d'intérêt des linguistes. Il serait aussi envisageable de réaliser des recherches autour de toutes les caractéristiques différenciatives que nous avons proposées. Nous espérons que ces aspects des gallicismes hispaniques et d'autres que nous n'avons pas pu évoquer, à cause du manque de place, seront l'objet de futures contributions.

\section{Références bibliographiques}

Asociación de Academias de la Lengua Española, 2010, Diccionario de americanismos, Lima, Santillana. (DASALE).

Baralt Rafael María, 1855, Diccionario de galicismos, Madrid, Imprenta Nacional.

Bohórquez Jorge, 1994, Concepto de 'americanismo' en la historia del español, Bogotá, Instituto Cano y Cuervo.

Cahuzac Philippe, 1988, «Approche méthodologique de l'étude des gallicismes en espagnol d'Amérique », in Hommage à Bernard Pottier, I, Paris, Klincksieck, p. 127-141.

Conde Óscar, 2004, Diccionario etimológico del lunfardo, Buenos Aires, Taurus. (DELOC).

Corbella Díaz Dolores, 1996/97, « Galicismos léxicos en el español de América ", Anuario de Lingüística Hispánica, 12/13, 2, p. 569-582.

Deroy Louis, 1956, L'emprunt linguistique, Paris, Société d'Édition «Les Belles Lettres".

Fontanella de Weinberg María Beatriz, 1986, El español bonaerense. Cuatro siglos de evolución lingüística, Buenos Aires, Hachette.

Garzón Tobías, 1910, Diccionario argentino, Barcelona, Imprenta Elzeviriana de Borrás y Mestres.

Granda Germán de, 1987-1989, «Dos episodios en el proceso de interferencia léxica del francés sobre el español dominicano (Toussaint l'ouverture, 1801 ; Boyer, 1822) » Estudios románicos, no 4 (Ejemplar dedicado a: Homenaje al profesor Luis Rubio (I), p. 537-546. 
Granda Germán de, 1990, « Galicismos léxicos en el español dominicano de la segunda mitad del siglo XVIII », Lexis, XIV, p. 197-219.

Haensch Günther, Werner Reinhold (éds.), 2000, Diccionario del español de Cuba. Español de Cuba / Español de España, Madrid, Gredos. (DECu).

Haensch Günther, Werner Reinhold (éds.), 2000, Diccionario del español de Argentina. Español de Argentina / Español de España, Madrid, Gredos. (DEAr).

Haensch Günther, Werner Reinhold (éds.), 1993, Nuevo Diccionario de Americanismos. Tomo I Nuevo Diccionario de Colombianismos, Bogotá, Instituto Caro y Cuervo. (DECo).

Haensch Günther, Werner Reinhold (éds.), 1993, Nuevo Diccionario de Americanismos. Tomo III. Nuevo Diccionario de Uruguayismos, Bogotá, Instituto Caro y Cuervo. (DEUr).

Haugen Einar, 1950, "The analysis of linguistic borrowing ", Language, 26, p. $210-231$.

Lara Ramos Luis Fernando (éd.), 1996, Diccionario del español usual en México. http://www.cervantesvirtual.com/obra-visor/diccionario-del-espanol-usual-en-mexico--0/html/ le 23.12.2016. (DEUMx).

Lope Blanch Juan Manuel, 1995, « Americanismo frente a españolismo lingüísticos ", Nueva revista de filología hispánica, 43, n 2, p. 433-440.

Moliner María, 2008, Diccionario de uso del español (cédérom), Madrid, Gredos. (DUE)

Plager Federico (coord.), 2008, Diccionario integral del español de la Argentina, Buenos Aires, Tinta Fresca - Voz Activa. (DIEA).

Pratt Comfort, 2004, El español del noroeste de Luisiana, Madrid, Verbum.

Prędota Stanisław, 2003, Wprowadzenie do językoznawstwa niderlandzkiego, Wrocław, Wydawnictwo Uniwersytetu Wrocławskiego.

Real Academia Española, 2014, Diccionario de la lengua española, http://www. rae.es/ le 12.12.2016. (DLE)

Rey-Debove Josette, 1973, «La sémantique de l'emprunt lexical», Travaux de linguistique et de littérature, XX/1, p. 109-124.

Romero Matías, 2005, Diccionario de salvadoreñismos, San Salvador, Delgado. (DEES).

Sala Marius, Munteanu Dan, Neagu Valeria, Sandru-Olteanu Tudora, 1982, El español de América, t. I, El léxico, parte primera, Bogotá, Instituto Caro y Cuervo.

Sorbet Piotr, 2014a, «La gastronomie ibérique dans la langue française », Revue d'Études Françaises, n 19 , p. 97-105. 


\section{Piotr SORBET}

Sorbet Piotr, 2014b, « Análisis lingüístico del vesre porteño », Roczniki Humanistyczne, LXII, p. 123-134.

Sorbet Piotr, 2015, «L'influence de la langue française sur l'espagnol argentin : les gallicismes lexicaux », Revue de la SAPFESU, n 38, p. 78-93.

Sorbet Piotr, 2016a, "Aproximación a la descripción lingüística del vesre porteño ", in Relecturas y nuevos horizontes en los estudios hispánicos, vol. 4: Lingüistica y didáctica de la lengua española,Wilk-Racięska Joanna, Szyndler Agnieszka, Tatoj Cecylia (éds.), p. 173-183.

Sorbet Piotr, 2016b, «En torno al tratamiento lexicográfico de los vesreísmos », Itinerarios, 23, p. 141-153.

Sorbet Piotr, 2016c, "Zapożyczanie leksykalizujące » in Badania diachroniczne w Polsce II. Między współczesnością a przeszłością, Krzyżanowska Anna, Posturzyńska-Bosko Małgorzata, Sorbet Piotr (éds.), Lublin, UMCS, p. 101-112.

Tagliavini Carlo, $1993^{2}$ [1973], Orígenes de las lenguas neolatinas : introducción a la filología romance, México, Fondo de Cultura Económica.

Tejera María Josefina, 19932, Diccionario de venezolanismos, Caracas, Academia Venezolana de la Lengua : Universidad Central de Venezuela. (DEVe).

Varela Merino Elena, 2009, Los galicismos en el español de los siglos XVI y XVII, Madrid, CSIC. 